Ann. Zootech., Ig69, 18 (3), 249-26I.

\title{
INFLUENCE DE LA TENEUR EN FER DES ALIMENTS D'ALLAITEMENT POUR VEAUX A L'ENGRAIS SUR LES ÉLÉMENTS FIGURÉS DU SANG, LA COULEUR DE LA VIANDE ET LES RÉSULTATS D'ENGRAISSEMENT ( $\left.{ }^{(}\right)$
}

\author{
W. EECKHOUT, M. CASTEELS ( $\left.{ }^{2}\right)$ et F. BUYSSE \\ avec la collaboration technique de Anny Van Hecke et Annie Lateur \\ Station de Recherches de l'État pour l'Alimentation du Bétail, \\ Gontrode (Belgique)
}

\section{SOMMAIRE}

$\mathrm{J}_{\mathrm{n}}$ aliment d'allaitement du commerce contenant $2 \mathrm{I}$ p.p.m. de fer a été distribué à un groupe de veaux à l'cngrais selon un plan de rationnement donné; en même temps 3 groupes analogues de veaux ont reçu le même aliment d'allaitement, mais supplémenté par des quantités croissantes de Fe sous form 2 de FeSO $\mathrm{F}_{4}-7 \mathrm{H}_{2} \mathrm{O}$, à raison de I 5 p.p.m., 30 p.p.m. et 45 p.p.m. calculées en Fe élémentaire.

Des échantillons de sang ont été analysés toutes les a semaines et leur teneur en hémoglobine ainsi que la valeur de l'hématocrite ont été déterminées.

En outre, il a été procédé à la fin de l'expérience à la numération des hématies. Les résultats mettent en évidence que la teneur en hémoglobine ct la valeur de l'hématocrite du sang continuent à baiss r jusqu'à la fin chez les veaux à l'engrais recevant un aliment d'allaitement normal pauvre en Fe. La supplémentation en $\mathrm{Fe}$, par contre, a exercé une influence favorable sur les éléments figurés du sang à partir de la 8 e semaine.

A la fin de l'expérience les veaux témoins étaient anémiques comparativement aux veaux qui avaient bénéficié d'une supplémentation en Fe. L'anémie était du type microcytaire normochrome. La teneur en pigment de la viande avait augmenté parallèlement aux doses de Fe administrées.

Cette administration de Fe par voie orale a favorablement influencé la croissance et l'efficacité alimentaire en dépit des conditions nettement défavorables de nos expériences, tant sous le rapport du traitement (prélèvements bi-mensuels de sang et pesées toutes les 4 semaines) que celui du logement (étable trop froide et humide).

\section{INTRODUCTION}

Le souci majeur des engraisseurs de veaux est la production "économique " de viande blanche. Or, on ne peut obtenir de " veau blanc " que si l'aliment d'allaite-

(') Communication $n^{0}$ I 77 de la Station de Recherches de l'État pour l'Alimentation du Bétail.

$\left({ }^{2}\right)$ Groupe de travail : Amélioration de la qualité de la viande bovine (Président. Pr. Dr. J. Martin). 
ment, l'eau utilisée et les conditions de stabulation préviennent l'assimilation de Fe aut-delà d'une quantité déterminée.

D'autre part il est prouvé (VERBEKE et MARTIN, I967) que le facteur " animal» exerce aussi une influence sur la couleur de la viande.

Les veaux qui abordent la période d'engraissement avec un sang à forte teneur en hémoglobine et une valeur de l'hématocrite élevée fourniront généralement une viande moins blanche, même en cas d'alimentation pauvre en fer, que les animaux plus anémiques dès le début de l'expérience.

Les fabricants d'aliments d'allaitement ont de lourdes exigences en ce qui concerne la teneur en fer des matières premières à employer et du produit fini. Par contre, les veaux peuvent contracter un degré d'anémie plus ou moins prononcé, ce qui influence défavorablement leur résistance naturelle. La pratique vétérinaire dès lors instaure très souvent un traitement aux préparations ferrugineuses lorsque des veaux sont en difficulté pendant la période d'engraissement. De même dans le passé où les veaux blancs étaient engraissés au lait entier et où l'ingestion de Fe provenant d'autres sources était minime, l'on constatait souvent de très faibles teneurs en hémoglobine, et ceci après environ 3 mois d'alimentation exclusive au lait entier.

Br.AXTER et al. ( 1967 ) ont provoqué l'anémie des veaux à l'engrais en appliquant exclusivement une alimentation lactée. Au cours des 6 premières semaines, la croissance était pratiquement identique à celle dess veaux qui, en plus du lait entier, recevaient du fer par voie orale et sous la forme de Ferricitrate. Au cours de la période suivante, les animaux traités au Fe manifestaient une nette tendance à une meilleure croissance et la différence de croissance après $\mathrm{I} 2$ semaines plaidait indiscutablement en faveur de la supplémentation en Fe. Rov et al. (I964) ont observé chez des veaux à l'engrais que l'injection intramusculaire de 3 fois $500 \mathrm{mg}$ de Fe sous la forme de Fe-dextrane exerçait une influence nettement positive sur la croissance, l'efficacité alimentaire et la teneur en hémoglobine du sang. Ce fer était administré, conjointement avec une alimentation au lait entier, les $7^{\mathrm{e}}, \mathrm{I} 4^{\mathrm{e}}$ et $2 \mathrm{I}^{\mathrm{e}}$ jouts, chaque fois à raison de $500 \mathrm{mg}$. Ce traitement n'exerçait pas seulement une influence favorable sur la croissance : il apparut que cette administration de Fe n'influençait guère la couleur de la viande. ORTH et DREWs (I G66) ont additionné resp. 50 et 200 p.p.m. Fe à un aliment d'allaitement déjà riche en Fe d'après nos normes ( 82 p.p.m.).

Des différences de croissance n'ont pas été enregistrées, mais la couleur de la viande était influencée négativement. Cirarpentrier (I966) a constaté que de fortes doses de $\mathrm{Fe}$ administrées par voie orale, rejpectivement $\mathrm{I} 5$, 30 et 45 jours avant l'abattage, sont en mesure d'intensifier nettement la couleur de la viande et de faire augmenter rapidement la valeur de l'hématocrite du sang. Des veaux qui avaient reçu les 15 derniers jours avant l'abattage enviton $20 \mathrm{~g}$ de Fe sous forme de sulfate de fer par voie orale, présentaient déjà une nette augmentation du fer héminique dans les muscles, ce qui impliquerait une incorporation du Fe à la myoglobine plutôt rapide à cet âge.

L'objectif des essais décrits ci-dessous était d'étudier l'influence du fer administré par voie orale, en l'incorporant à un aliment d'allaitement, en concentrations compatibles avec les possibilités de la pratique et permettant de se prononcer sur le degré et la forme d'anémie établie chez les veaux à l'engrais dans les conditions normales.

Un autre objectif était de fixer une limite maximale admissible à la teneur en Fe 
des aliments d'allaitement pour veaux à l'engrais afin de produire encore des veaux à viande d'une couleur acceptable.

Les données de croissance et de consommation d'aliments nous apprendraient si une supplémentation en Fe est souhaitable.

\section{MATÉRIEI, E'T MÉTHODES}

\section{Matériel animal, logement et traitement}

Trente-deux veaux de la race Pie rouge de la Flandre orientale ont été utilisés. Il s'agissait cle 28 veaux mâles et de 4 veaux femelles. Les animaux furent répartis en 4 groupes homogènes, compte tenu du sexe, du poids, de la teneur en hémoglobine et de la valeur de l'hématocrite de leur sang.

Les teneurs en hémoglobine et les valeurs de l'hématocrite furent portées au même niveau dès le début de l'expérience, non seulement pour permettre une meilleure comparaison de l'évolution de ces paramètres sous l'influence de doses croissantes de $\mathrm{Fe}$, mais aussi pour éliminer autant que possible l'influence probable de la teneur en hémoglobine et la valeur de l'hématocrite initiales sur la couleur de la viande (Verbeke et Martin, 1967; Charpentier, ig66; Casteels et Eeckhout, résultats non publiés).

Un veau mâle du groupe $n^{\circ} 3$ mourut au bout de deux semaines, réduisant ce groupe à 7 animaux. Le tableau $\mathbf{I}$ donne un aperçu du matériel animal utilisé, par groupe expérimental, au début de l'expérience.

\section{TABLEAU I}

Sexe, poids initial, teneur en hémoglobine du sang et valcur de l'hématocrite

\begin{tabular}{|c|c|c|c|c|c|c|}
\hline \multirow{2}{*}{ Groupe expérimental } & \multirow{2}{*}{$\begin{array}{c}\text { Fe total } \\
\text { dans l'aliment } \\
\text { d'allaitement } \\
\text { (p.p.m.) }\end{array}$} & \multicolumn{2}{|c|}{ Sexe } & \multirow{2}{*}{$\begin{array}{l}\text { Poids } \\
\text { (en kg) }\end{array}$} & \multirow{2}{*}{$\begin{array}{l}\text { Héno- } \\
\text { globine } \\
(\mathrm{g} / 100 \mathrm{ml})\end{array}$} & \multirow{2}{*}{$\begin{array}{l}\text { Valeur de } \\
\text { l'hérnato- } \\
\text { crite }\end{array}$} \\
\hline & & Femelle & Mâle & & & \\
\hline Témoin ............ & 21 & 1 & 7 & 47,75 & 12,1 & 42,3 \\
\hline+15 p.p.m. le.... & 36 & 1 & 7 & 47,38 & 12,8 & 43,2 \\
\hline+30 p.p.m. le... & 51 & 1 & i & 47,86 & 12,8 & 14,6 \\
\hline$+{ }^{4} 5$ p.p.m. Fe... & 66 & 1 & 7 & 47,25 & 12,5 & 43,1 \\
\hline
\end{tabular}

Les animaux étaient logés dans des boxes en bois, comme c'est l'usage pour les veaux à l'engrais. Les boxes ne comportaient aucun élément de construction en fer. L'expérience a toutefois débuté l'hiver et le climat de l'étable était défavorable : humidité excessive et température trop basse (environ $\mathrm{r}^{\circ} \mathrm{C}$ ). Les animaux étaient retirés de leurs boxes tous les $\mathrm{I}_{4}$ jours pour un prélèvement de sang. Ils étaient pesés toutes les 4 semaines.

\section{Aliment d'allaitement}

L'aliment d'allaitement contenait environ i 8 p. Ioo de matières grasses, 23 p. Ioo de protéines brutes totales, dont $22,5 \mathrm{p}$. Ioo digestibles et $50 \mathrm{~g}$ d'antibiotique par tonne. La teneur en fer était de 2 I p.p.m. On y ajouta:

pour le groupe expérimental B : I5 p.p.m. de Fe pour le groupe expérimental $\mathrm{C}: 3^{\circ}$ p.p.m. de $\mathrm{Fe}$ pour le grouge expérimental D : 45 p.p.m. de Fe toujours sous forme de $\mathrm{FeSO}_{4}-7 \mathrm{H}_{2} \mathrm{O}$. 
Les repas étaient servis 2 fois par jour conformément au schéma de la figure $\mathbf{r}$. La teneur en Fe de l'eau utilisée pour la dissolution de la poudre de lait était en moyenne de 0,65 p.p.m.

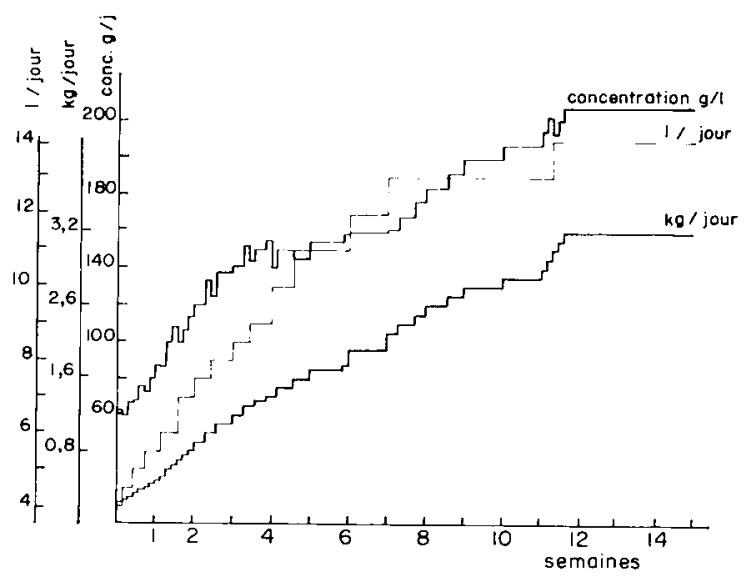

FiG. I. - Plan de rationnement

Méthodes

Le sanor prélevé à la veine jugulaire a été recueilli dans de l'héparine. L'hémoglobine a été déterminée par la méthode de l'hématine alcaline et la valeur de l'hématocrite après 30 minutes de centrifugation à $3500 \mathrm{t} / \mathrm{m}$ en tubes de Wintrobe. I.es érythrocytes ont été comptés en cellules hématimétriques de Burker après dilution au centuple dans le liquide de Hayem. La technique décrite par Hokxsey (1956) a été appliquée pour déterminer post-mortem la teneur en pigment d'échantillons le Longissimus dorsi et de Latissimus dorsi.

Pour cléterminer la teneur en Fe du lobe gauche lu foie nous avons, après lavage à l'eau distillée du sang superficicl, séché et soigneusement moulu les tranches de foic. Les déterminations du fer de cette farine de foie ainsi que de l'aliment d'allaitement et clu résidu de dessiccation de l'eau utilisón pour la préparation du lait de remplacement ont été effectuées après minéralisation humicle à l'aide du mélange d'acides de I’Yck et al. (I958).

Nous aroni toujours employé ro $\mathrm{ml}$ de mélange d'acicles pour la destruction de $\mathrm{I} g$ de farine de foie, I g d'aliment d'allaitement ou du résidu sec de roo $\mathrm{ml} \mathrm{d'eau.}$

Après transwasement de la solution minéralisée dans des ballons jaugés et dilution aqueuse jusqu'à $50 \mathrm{ml}$, la détermination du fer a cu lieu sur une fraction de $\mathrm{l}$ o $\mathrm{ml}$ par la méthode à l'orthophénanthroline de BANDEMkr et SCHAIBLE (1944). Après l'addition de tous les réactifs et la dilution, on attendait cependant le matin suivant avant de procéder aux mesures spectrophotométriques.

\section{RÉSULTATS ETT DISCUSSION}

\section{I. Évolution de l'hémoglobine et de l'hématocrite en fonction de la durée de l'engraissement}

L'influence exercée sur la valeur de l'hématocrite et sur la teneur en hémoglobine par le fer administré par voie orale, par l'intermédiaire de l'aliment a été étudiée sur les échantillons de sang prélevés toutes les 2 semaines. Le sang prélevé à partir de la veine jugulaire était recueilli dans des éprouvettes contenant de l'héparine comme anticoagulant. Le prélèvement du sang (env. ro $\mathrm{ml}$ ) avait toujours lieu le matin, avant le premier repas. 
A cette fin, il fallait sortir les veaux de leurs boxes, opération qui créait un certain état de stress, lequel a probablement influencé les teneurs en hémoglobine et les valeurs de l'hématocrite. On sait, en effet, que l'excitation, la peur et un gros effort font monter ces valeurs à des niveaux anormalement élevés (ARCHER, I965 ; GREATOREX, I954). Il est cependant impossible d'éliminer toutes les formes de stress dans les conditions régnant dans la pratique.

Le tableau 2 et les figures 2 et 3 donnent un aperçu de l'évolution de la valeur de 1'hématocrite et de la teneur en hémoglobine du sang en fonction de la durée de l'engraissement et des différentes doses de fer administrées. Les différents niveaux de $\mathrm{Fe}$ de l'aliment d'allaitement n'ont eu, au cours des 6 premières semaines de l'expérience, que peu ou pas d'influence sur la teneur en hémoglobine et sur la valeur de l'hématocrite du sang.

\section{TABLEAU 2}

Influence do la duvée de l'engraissemont et de la teneur en $F e$ de l'aliment d'allaitement sur l'hémoglobine du sang ct sur la valeur de l'hématocrite chez les veaux à l'engrais

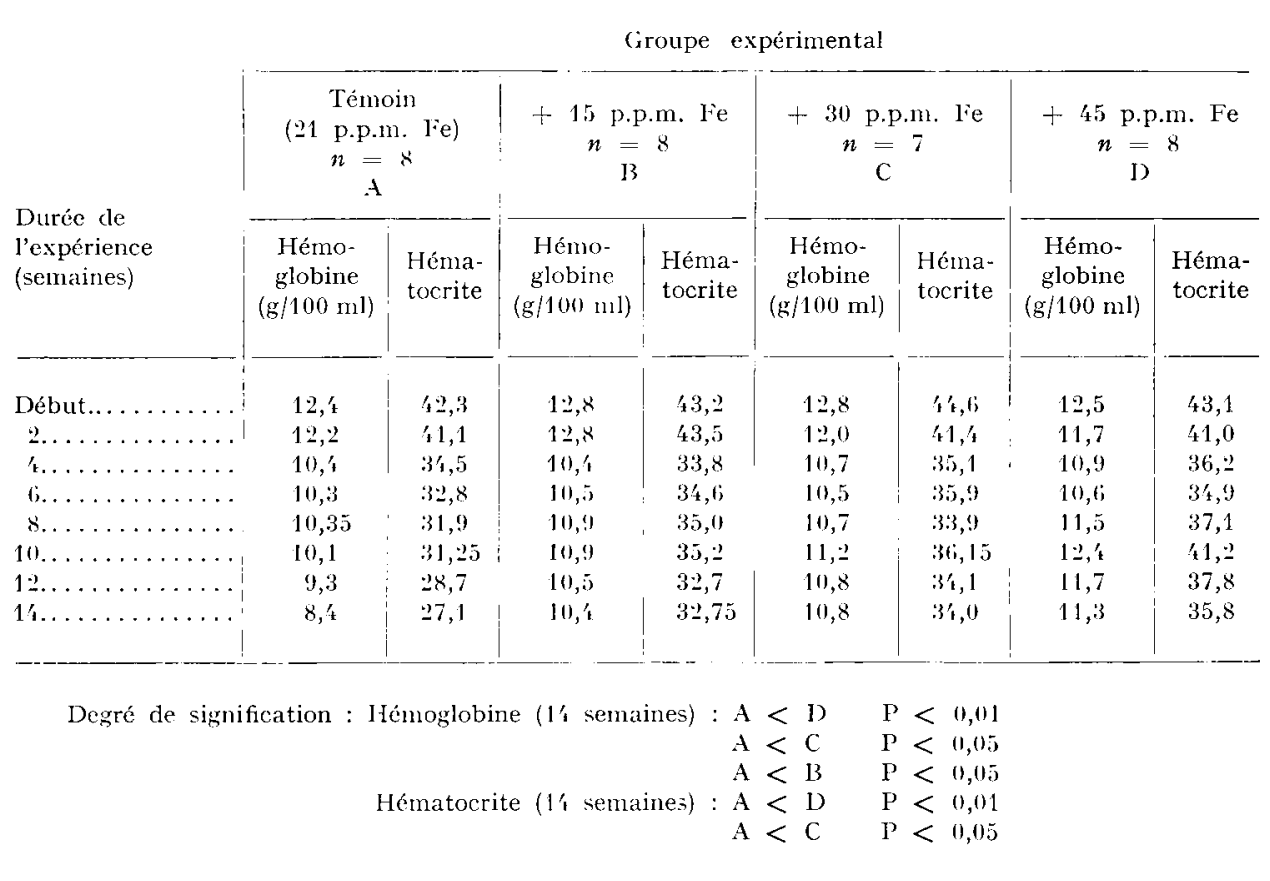

L,es aliments d'allaitement supplémentés en Fe et l'aliment d'allaitement témoin ont provoqué une baisse analogue. Une différenciation de la teneur en hémoglobine et de la valeur de l'hématocrite du sang, influencée par les différentes doses de Fe, est devenue notable à partir de la $8^{\mathrm{e}}$ semaine d'expérience et s'est accentuée à mesure que l'engraissement se poursuivait.

Alors que la teneur en hémoglobine et la valeur de l'hématocrite manifestaient

Annales de Zootechnie. - I 969. 
une baisse évidente et continue chez les veaux recevant 1'aliment d'allaitement témoin (le plus pauvre en $\mathrm{Fe}$ ) - constatation conforme aux données de SchmrtTEN et NoAck (I967) et de Verbeke et MarTrs ( 1967 ) - ces valeurs se maintenaient plutôt au même niveau chez les veaux recevant du Fe supplémentaire via 1'aliment d'allaitement. Une tenđance nettement ascendante fut même observée chez les veaux recevant l'aliment additionné de 45 p.p.m. de Fe. Le pic des courbes d'hémoglobine et d'hématocrite à Io semaines est frappant pour les deux plus fortes doses de supplémentation en fer. (Ces effets de pic ne sont pas le résultat de quelques chiffres élevés relevés à ce moment chez un couple de veaux.)

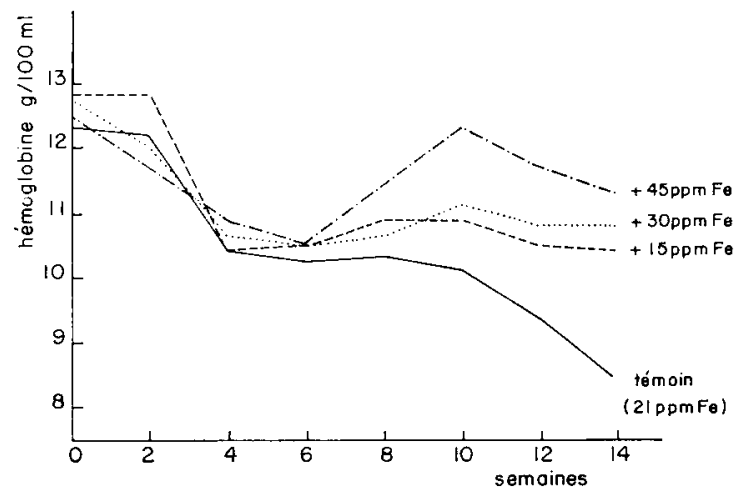

FIG. 2. - Evolution de la concentration en hémoglobine

en fonction de la durée de l'engraissement et de la teneur en fer de l'aliment d'allaitement

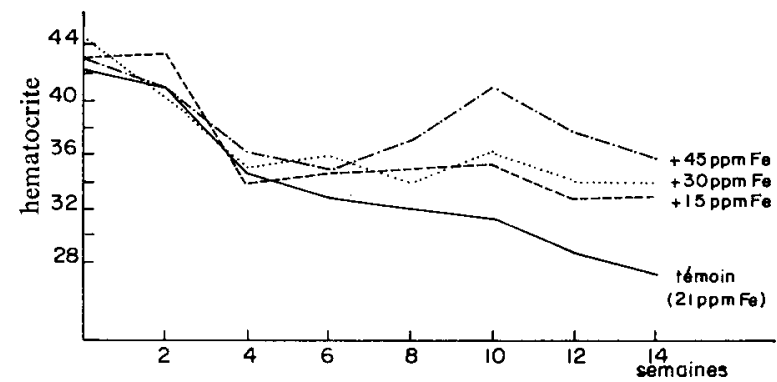

FIG. 3. - Evolution de l'hématocrite en fonction de la durée de l'engraissement et de la teneur en fer de l'aliment d'allaitement

Les courbes d'hémoglobine et d'hématocrite des veaux recevant les doses supplémentaires de $\mathrm{Fe}$ en cause manifestent pratiquement toutes cet effet de pic à cet âge (Io semaines). Les observations de FOW IER et BARBER (I94I) sont intéressantes sous ce rapport.

Ces auteurs ont relevé chez l'homme, une augmentation de la teneur en hémoglobine du sang consécutive à un traitement au fer, avec un maximum après ro à I2 semaines d'administration de fer, augmentation suivie d'un retour progressif à la teneur en hémoglobine normale, ceci indépendamment de la poursuite ou de l'interruption du traitement au fer. La baisse qui se manifeste dans nos essais après le pic à 
Io semaines est toutefois telle que l'influence de l'administration de Fe subsiste nettement à I4 semaines. Les teneurs en hémoglobine et les valeurs de l'hématocrite atteintes sont proportionnelles à la dose de fer administrée, sans toutefois que les valeurs initiales présentes au début de l'expérience aient été égalées.

2. Infuence de divers niveaux de Fe sur les éléments figurés du sang des veaux à la fin de l'expérience (environ II2 jours d'expérience)

Un prélèvement de sang de la veine jugulaire a été effectué à la fin de l'expérience, le matin du jour d'abattage, d'une manière analogue à celle pratiquée au cours de la période d'engraissement. En plus de la détermination de la teneur en hémoglobine et de la valeur de 1'hématocrite, nous avons procédé cette fois, au comptage des érythrocytes. Nous en avons déduit : le volume moyen des érythrocytes, la teneur moyenne en hémoglobine des cellules ainsi que leur concentration moyenne en hémoglobine. Le tableau 3 donne un aperçu des résultats obtenus.

\section{TABLEAU 3}

Influence de différentes doses de Fe sur les éléments figurés du sang de veaux à l'engrais, p la fin de l'expérience (environ $\mathrm{I} \mathbf{2} 2$ jours d'expérience)

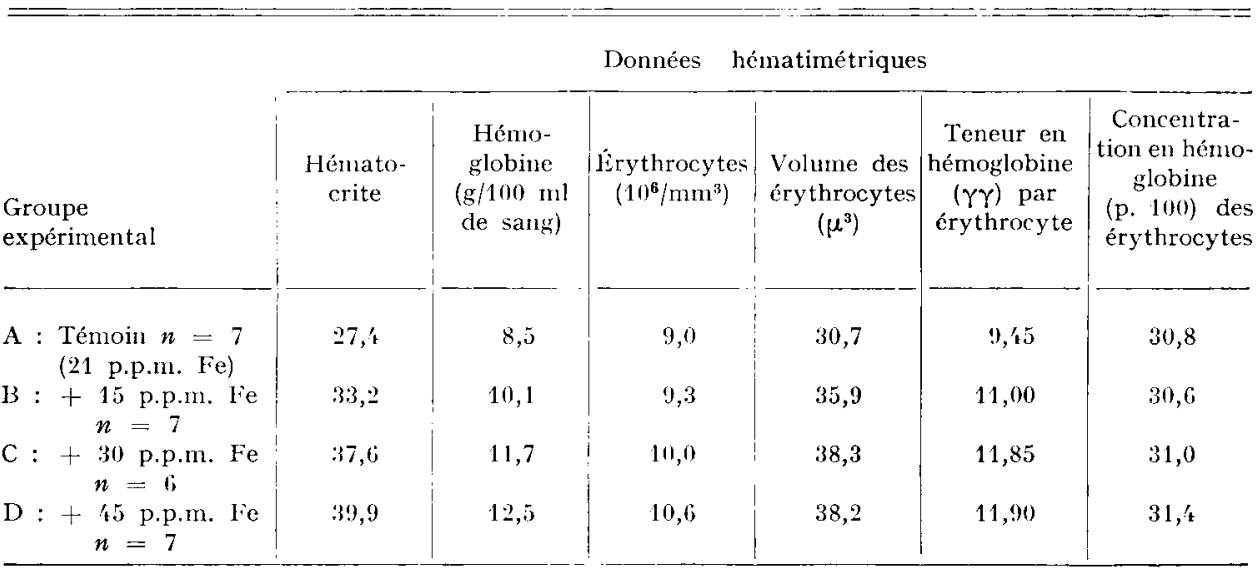

Degré de signification : Hématocrite (final) $: A<D$

$\mathrm{A}<\mathrm{C} \quad \mathrm{P}<0,01$

$\mathrm{B}<\mathrm{D} \quad \mathrm{P}<0,05$

Hémoglobine (finale) : $A<B \quad P<0,01$

$\mathrm{A}<\mathrm{C} \quad \mathrm{P}<0,01$

$A<\mathrm{D} \quad \mathrm{P}<0,01$

$\mathrm{B}<\mathrm{C} \quad \mathrm{P}<0,01$

$\mathrm{B}<\mathrm{D} \quad \mathrm{P}<0,01$

Erythrocytes $\left(10^{6} / \mathrm{mm}^{3}\right):$ NS

$\begin{array}{rlrl}\text { Volume des érythrocytes }\left(\mu^{3}\right): & A<D & P<0,01 \\ & A<C & P<0,01 \\ \text { Teneur en hémoglobine }(\gamma \gamma): A<D & P<0,05 \\ & A<C & P<0,05\end{array}$


Le Fe supplémentant l'aliment d'allaitement a exercé une influence notable sur les données hématologiques obtenues. La valeur de l'hématocrite de même que la teneur en hémoglobine ont été influencées positivement par les doses croissantes de Fe. On observe en outre une augmentation du nombre de globules rouges par unité de volume de sang. Il découle de la combinaison de ces données que le volume moyen des érythrocytes, exprimé en microns cubes, augmente sous l'influence des doses croissantes de $\mathrm{Fe}$, de même que leur teneur moyenne en hémoglobine (exprimée en microgrammes d'hémoglobine).

La concentration en hémoglobine dans les érythrocytes semble, par contre, n'avoir été que peu influencée par les différentes administrations de Fe.

On peut donc en conclure que les veaux recevant un aliment d'allaitement pauvre en fer sont anémiques par rapport à ceux recevant un aliment d'allaitement enrichi en fer et que l'anémie observée est du type microcytaire normochrome.

Le nombre de globules rouges par unité de volume n'est pas seulement plus petit, sous l'influence d'un aliment d'allaitement pauvre en fer, que chez des veaux recevant un aliment d'allaitement plus riche en fer ; ces globules sont aussi plus petits alors que la concentration en hémoglobine du globule reste normale.

Le nombre élevé d'érythrocytes, associé à un volume moindre des globules est à l'origine des faibles valeurs de l'hématocrite relevées chez les veaux témoins. La concentration en hémoglobine du globule restant, en outre, inchangée, la teneur en hémoglobine du sang diminue forcément. Ia bibliographie rapporte cependant que des teneurs en hémoglobine et des valeurs de l'hématocrite encore plus faibles ont été relevées dans le cas d'alimentation exclusive au lait entier ò̀, de plus, l'ingestion de Fe du milieu était négligeable.

De fait, le lait entier n'a qu'une très faible teneur en fer, à peine de l'ordre de 0,5 p.p.m. Le lait ayant une teneur en matière sèche d'environ I 2 p. Ioo, la matière sèche de l'aliment d'allaitement contient une quantité relativement beaucoup plus élevée de fer (de l'ordre de 2 I p.p.m.) que celle du lait entier (de l'ordre de 4 à 5 p.p.m.)

BlAXTER et al. (I957) ont relevé après I2 semaines d'alimentation exclusive au lait entier une teneur en hémoglobine de $4,9 \mathrm{~g} /$ Ioo $\mathrm{ml}$ et une valeur de l'hématocrite de 15,2 . Ces valeurs s'élevaient toutefoi à respectivement 7,9 et $27,7 \mathrm{~g} / \mathrm{I} 00 \mathrm{ml}$ quand on supplémentait quotidiennement, par voie orale, le lait entier de $20 \mathrm{mg}$ de Fe. D'autres expériences effectuées en Grande-Bretagne (Roy et al., I964) ont aussi permis de mettre en évidence que l'alimentation exclusive au lait entier aboutit à des teneurs en hémoglobine et des valeurs de l'hématocrite faibles, notamment $5,2 \mathrm{~g} / \mathrm{I} 00 \mathrm{ml}$ d'hémoglobine et une valeur de l'hématocrite de 20,4 .

En revanche, quand on administrait aux animaux 3 fois $500 \mathrm{mg}$ de Fe sous forme de Fe-dextrane, en injections intramusculaires et en 3 doses égales étalées sur les 3 premières semaines d'expérience, la teneur en hémoglobine et la valeur de l'hématocrite atteignaient respectivement $8,2 \mathrm{~g} / \mathrm{I} 00 \mathrm{ml}$ et 27,4 , c'est-à-dire des niveaux nettement plus élevés que chez les veaux témoins du même âge ( 12 semaines).Tant dans les expériences de BlaxTER et al. (I957) que dans celles de Roy et al. (I964) l'administration de Fe a exercé une influence nettement favorable sur la croissance. YEates (Ig66) n'a observé aucune diminution de la teneur en hémoglobine, mais en constatait une pour la valeur de l'hématocrite du sang des veaux pendant les 4 premiers mois ; cependant ces animaux ne recevaient aucun autre aliment que le lait frais de 
vaches " nourrices". Ces veaux étaient toutefois logés sur une aire en béton et disposaient de sel distribué sous forme de pierres à lécher. En outre ils rejoignaient 2 fois par jour les vaches nourrices à l'herbage. De plus, aucune précaution n'était prise pour empêcher l'ingestion de fer provenant du milieu. De roo à $\mathrm{I}_{5} \mathrm{O} \mathrm{g}$ de sable furent trouvés dans le rumen des animaux abattus. On peut supposer que le maintien de l'hémoglobine à son niveau initial est imputable à une ingestion plus considérable de fer provenant du milieu, quoique d'autres oligo-éléments ingérés, tels que le cuivre et le cobalt, puissent aussi en être partiellement responsables.

\section{Influence de l'administration de fer sur la couleur de la viande et sur la tenour en fer du foie}

Les animaux ont été abattus le mardi à une semaine d'intervalle. Une partie du foie (le lobe gauche) fut découpée après l'abattage et emportée au laboratoire en vue de la détermination du Fe.

Les carcasses furent découpées le lendemain de l'abattage et la "blancheur " du Longissimus dorsi mesurée à l'aide d'un Fahellpho Mariensee - LoHsE et PFAU (I964). Un échantillon de viande contenant e. a. le Longissimus dorsi et le Latissimus dorsi, prélevé à la hauteur de la $5^{\mathrm{e}}$ côte, fut également emporté au laboratoire en vue de la détermination de la teneur en pigment.

Le tableau 4 ci-dessous donne un aperçu des résultats obtenus quant à la couleur de la viande et la teneur en $\mathrm{Fe}$ du foie.

\section{TABLEAU 4}

Influence de différentes doses de Fe sur la couleur de la viande et sur la teneur en Fe du foie.

\begin{tabular}{|c|c|c|c|c|}
\hline \multirow{2}{*}{ Groupe expérimental } & \multirow{2}{*}{$\begin{array}{l}\text { Fahellpho } \\
\text { (indice de } \\
\text { blancheur) } \\
\text { Long. dorsi }\end{array}$} & \multicolumn{2}{|c|}{$\begin{array}{l}\text { Hématine (p.p.m.) } \\
\text { (indice de teneur en pigment) }\end{array}$} & \multirow{2}{*}{$\begin{array}{l}\text { Teneur en fer } \\
\text { du foie (parg } \\
\text { de produit frais) }\end{array}$} \\
\hline & & Long. dorsi & Lat. dorsi & \\
\hline $\begin{array}{c}\text { A : Témoin } n=7 \\
(21 \text { p.p.n. Fe })\end{array}$ & 78,2 & $3^{\prime}, 70$ & 48,3 & 21 \\
\hline $\begin{array}{c}\text { B : }+15 \text { p.p.m. Fe } \\
n=7\end{array}$ & 69,8 & 43,50 & 53,0 & 20 \\
\hline $\begin{array}{c}\mathrm{C}:+30 \text { p.p.m. Fe } \\
n=6\end{array}$ & 63,0 & 16,25 & 56,5 & 27 \\
\hline 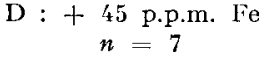 & 61,4 & 58,50 & 70,0 & 25 \\
\hline
\end{tabular}

Degré de signification : Fahellpho: $A>D \quad P<0,01$

$$
A>C \quad P<0,05
$$

Hématine :

(Long. dorsi) $\mathrm{A}<\mathrm{D} \quad \mathrm{P}<0,05$

(Lat. dorsi) $\mathrm{A}<\mathrm{D} \quad \mathrm{P}<0,05$

Teneur en Fe du foie : NS. 
Comme l'indiquent les valeurs Fahellpho décroissantes, la "blancheur " du Longissimus dorsi diminue sous l'influence de quantités croissantes de Fe dans l'aliment d'allaitement. On observe de même une augmentation progressive de la teneur en pigment des deux muscles examinés. Une plus grande teneur en Fe du foie a été enregistrée pour les 2 plus fortes doses de supplémentation en $\mathrm{Fe}$.

\section{I'infuence de l'administration de Fe sur la croissance et sur l'efficacité alimentaire}

ROY et al. (I964) et BLAXTHR (I957) ayant enregistré des résultats positifs en matière de croissance et d'efficacité alimentaire chez des veaux recevant, en plus de lait entier, une supplémentation en $\mathrm{Fe}$, il nous a paru intéressant d'examiner aussi l'influence de la supplémentation en Fe sur la croissance et sur l'efficacité alimentaire au cours de nos expériences utilisant l'aliment d'allaitement (tab1. 5).

TABLEAL 5

Résultats de croissance et efficacité alimentaires

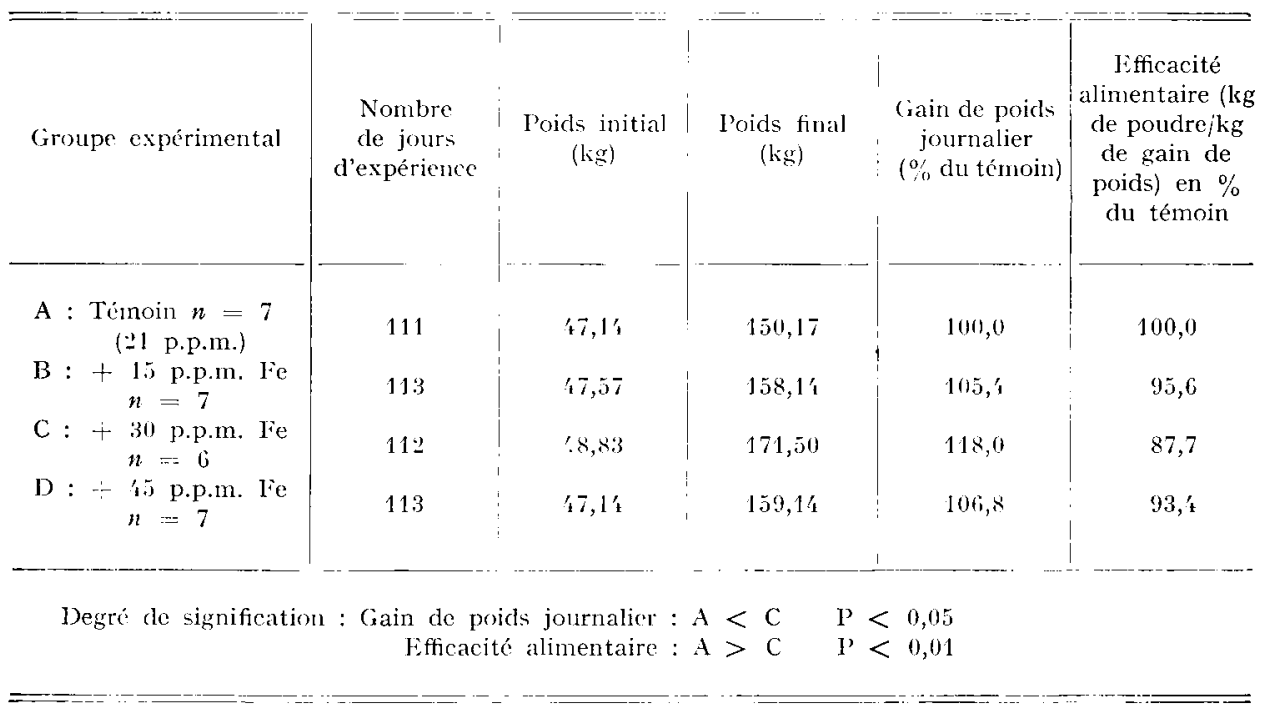

En général la croissance et l'efficacité alimentaire se sont révélées défavorables dans cette expérience. Comme nous l'avons dit d'emblée, le climat de l'étable (température et humidité) à l'époque de l'expérience (hiver) était loin d'être optimal. A ceci s'ajoute le dérangement causé par le prélèvement bi-mensuel de sang, et sans doute aussi la pesée mensuelle ce qui risquait de modifier la valeur de l'hématocrite et le taux d'hémoglobine.

Le tableat 5 fait néanmoins apparaître un effet favorable de l'administration de fer sur la croissance et sur l'efficacité alimentaire, ceci pour tous les groupes expérimentaux qui ont reçu les aliments d'allaitement supplémentés en Fe. Les résultats du groupe expérimental $\mathrm{C}\left(+3^{\circ}\right.$ p.p.m. de Fe étaient nettement les meilleurs. Le 
gain de poids fut supérieur de I 8 p. Ioo et l'efficacité alimentaire plus avantageuse de I 2,3 p. Ioo comparativement aux résultats des veaux témoins. Les gains en ce qui concerne à la fois la croissance et l'efficacité alimentaire se sont situés aux environs de 6 p. roo pour les groupes expérimentaux B (+ I5 p.p.m. de Fe) et D ( + 45 p.p.m. de $\mathrm{Fe}$ ).

Si ces chiffres plaident en faveur d'une augmentation de la teneur en Fe de l'aliment d'allaitement pour veaux à l'engrais, il convient d'avancer quelques considérations importantes. En premier lieu, il résulte inévitablement d'une plus forte teneur en $\mathrm{Fe}$, une viande moins blanche et plus riche en pigment (viande plus foncée). Fin second lieu, il est possible que les avantages constatés quant à la croissance et à l'efficacité alimentaire n'apparaissent que dans des circonstances défavorables. Nous entendons par là : climat d'étable moins bon, dérangements fréquents des veaux, pesées, prélèrement de sang, stress, etc.

SETTLEMIRE et al. (I964) ont d'ailleurs enregistré un pouls significativement plus rapide chez des veaux anémiques comparés à des animaux au sang plus riche en hémoglobine.

BL.AXTER et al. (r957) ont enregistré chez de; veaux anémiques, après excitation, un pouls de plus de $25^{\circ}$ à la minute, et qui ne revenait pas à la normale au bout de 40 minutes de récupération ; en revanche, les animaux témoins réagissaient à l'excitation par un pouls atteignant au maximum 200 à la minute et revenant à la normale au bout de 20 minutes de repos. C'est pourquoi il sera nécessaire de répéter une expérience analogue à celle décrite ici, mais dans laquelle tous les facteurs de perturbation possibles seront éliminés.

\section{CONCLUSIONS}

Les résultats décrits ci-dessus, mettent en évidence une anémie de plus en plus prononcée des veaux au cours de l'engraissement ; la teneur en hémoglobine comme la valeur de l'hématocrite du sang manifestent une évolution décroissante. En fin de compte il en résulte chez l'animal prêt pour l'abattage un état d'anémie, pourtant moins grave avec les aliments d'allaitement qu'avec exclusivement du lait entier, les conditions de logement étant comparables (Roy et al., I964). Ceci est peut-être imputable à une plus forte teneur en Fe de l'aliment d'allaitement comparativement au lait entier. Par rapport au sang de veaux qui avaient reçu le même aliment d'allaitement et un supplément de $\mathrm{Fe}$ sous forme de $\mathrm{FeSO}_{4}-7 \mathrm{H}_{2} \mathrm{O}$ (addition de respectivement I 5,30 et 45 p.p.m. de Fe, à un aliment d'allaitement contenant déjà $2 \mathrm{I}$ p.p.m. de $\mathrm{Fe}$ ), le sang des animaux n'ayant reçu aucune supplémentation en Fe présentait une valeur de l'hématocrite inférieure, une plus faible teneur en hémoglobine et un plus petit nombre d'érythrocytes au $\mathrm{mm}^{3}$, avec un volume moindre des globules rouges, mais avec une concentration en hémoglobine globulaire comparable. Les veaux nourris avec un aliment d'allaitement pauvre en fer présentaient de ce fait, à la fin de la période d'engraissement, un état d'anémie microcytaire normochrome.

Les symptômes d'anémie disparaissaient à mesure que la teneur en Fe de l'aliment d'allaitement augmentait et parallèlement à la dose de $\mathrm{Fe}$ administrée. Une plus forte teneur en $\mathrm{Fe}$ a, en outre, été relevée dans les foies des 2 groupes recevant 
les plus fortes supplémentations. D'après le tracé des courbes d'hématocrite et d'hémoglobine, l'addition de 45 p.p.m. de $\mathrm{Fe}$ au lait reconstitué n'a pas été en mesure de contrarier la diminution de la teneur en hémoglobine et de la valeur de l'hématocrite. L'effet de la supplémentation en Fe ne se manifestait qu'après 6 semaines par un tracé ascendant des courbes d'hémoglobine et d'hématocrite. L'influence de quantités croissantes de Fe dans l'aliment d'allaitement a eu un effet négatif sur la couleur de la viande. Il nous semble donc que la teneur en Fe des aliments d'allaitement pour veaux à l'engrais ne devrait pas dépasser 30 p.p.m. et qu'il serait même plus sûr de la maintenir aux environs de 20 p.p.m. si l'on attache beaucoup d'importance à l'obtention de veaux blancs. Il est encore possible d'obtenir une viande de veau d'une blancheur acceptable en utilisant un aliment d'allaitement contenant 30 p.p.m. de Fe en veillant toutefois à ce que les conditions suivantes soient réalisées : très faible teneur en fer de l'eau, état de logement empêchant l'ingestion de fer et, éventuellement, d'autres oligo-éléments, engraissement de veaux ne présentant au départ que des teneurs en hémoglobine et des valeurs de l'hématocrite du sang faibles (VERBEkE et Mar'tin, I967 ; Charpentier, I966). L'eau que nous avons employée pour préparer le breuvage lacté titrait 0,65 p.p.m. de Fe.

Il ressort de la bibliographie que la supplémentation en Fe du lait entier exerce effectivement une influence favorable sur la croissance, surtout à partir de Io semaines. Il faut toutefois souligner qu'à cet âge les veaux se trouvent déjà dans un état d'anémie plus avancée que ceux recevant des aliments d'allaitement. Nos expériences ont aussi fait apparaître un effet favorable de la supplémentation en Fe sur la croissace et sur l'efficacité alimentaire.

Nous désirons néanmoins être prudents en formulant ces conclusions car ces expériences se sont déroulées dans des conditions défavorables en ce qui concerne le climat de 1'étable, la manipulation des veaux (prélèvement de sang bi-mensuels). Ces chiffres pourraient cependant signifier que la capacité de réaction des animaux aux conditions de logement et aux traitements défavorables s'améliorent au fur et à mesure que leur état d'anémie s'atténue. La croissance et l'efficacité alimentaire plus favorables des animaux traités au Fe en seraient alors une conséquence.

(Traduit du néerlandais.)

Reçu pour publication en juin 1969.

\section{SUMMARY}

\section{INFLUENCE OF VARIOUS AMOUNTS OF IRON IN MILK REPLACERS \\ ON BLOOD CONSTITUENTS, MEAT COLOR \\ AND FATTENING RESUITS IN VEAI CAIVES}

Four milk replacers for veal calves were studied which had identical composition but differed in Fe content. The amounts of Fe studied were :
A) 2 I p.p.m. ;
B) 36 p.p.m. (2I p.p.m. + I5 p.p.m. of $\mathrm{Fe}$ as $\mathrm{FeSO}_{4} \cdot 7 \mathrm{H}_{2} \mathrm{O}$ );
C) $5 \mathrm{I}$ p.p.m. (2I p.p.m. + 30 p.p.m. of $\mathrm{Fe}$ as $\mathrm{FeSO}_{4} \cdot 7 \mathrm{H}_{2} \mathrm{O}$ ) ;
D) 66 p.p.m. (2I p.p.m. +45 p.p.m. of $\mathrm{Fe}$ as $\mathrm{FeSO}_{4} \cdot 7 \mathrm{H}_{2} \mathrm{O}$ ). 
The influence of the iron content in milk replacers on the evolution of blood hemoglobin content (see fig. 2 and table 2) and the hematocrite value (see fig. 3 and table 2) was studing during a $I_{4}$ weeks experimental period on 4 groups of veal calves.

In all veal calves a comparable drop in hematocrite value and hemoglobin content was observed up to the end of the 6 th week of the experiment. This was independent of the iron doses administered. A differenciation in terms of the iron received was next noted. The more iron the milk contained, the higher were the hematocrite value and hemoglobin content at the end of the fattening period.

A pronounced peak was recorded at the end of the Ioth week in the hemoglobin and in the hematocrite graphs of the group D $(2 \mathrm{I}+45$ p.p.m. I $)$. A blood sample was taken from each calf at the end of the experiment ( \pm I I 2 days) and the erythrocytes counted.

The hematocrite value and hemoglobin content were also determined at that time. The mean corpuscular volume $\left(\mu^{3}\right)$, the mean corpuscular hemoglobin content $(\gamma \gamma)$, and the mean corpuscular hemoglobin concentration (p. Ioo) were calculated using these data. The results are shown in table 3. They indicate that the control calves. fed a milk replacer poor in iron developed an anemia of the microcytic normochromic type. Increased iron content in milk replacers may avoid this state of anemia.

However, this increased content affects meat color. The Longissimus dorsi and the Latissimus dorsi have an increased hematin content (HOTRNSEY, I956). There is an increase in liver iron content, but it is not significant (see table 4 ).

The results of fattening show the influence of iron supplementation in milk replacers. Weight gain and feed efficiency are more favorable in groups B, C, and D than in the control calves (table 5).

\section{RÉFÉRENCES BIBLIOGRAPHIQUES}

ARCHER R. K., 1965. Haematological techniques for use on aninals. Blackwell, Oxford.

Bandemer, Selma L., Schaible P. J., I944. Determination of iron. A study of the o-phenanthroline method. Ind. Eng. Chem. (Anal. Ed.), 16,317.

Blaxter K. L., Sharman G. A. M., MacDonald A. M., 1957. Iron-deficiency anemia in calves. Br. J. Nutr., 11, 234 .

Charpentier J., rg66. Pigmentation musculaire du veau de boucherie. II. Influence d'une supplémentation alimentaire en fer sur la teneur en fer héminique de quelques muscles. Ann. Zootech., 15, 136.

Fowler W. M., Barer A. P., I94I. Amer. Med. Sci, 210, 642. Citation extraite de Iron Metabolism Gross, F. Springer-Verlag Berlin (1964).

Greatorex J. C., I954. Studies on the hematology of calves from birth to one year of age. Br. Vet. J., 110, I20.

Hornsey H., I956. The colour of cooked cured pork. J. Sci. Food Agric., $7,534$.

Lohse B., Prau A., I964. Ein handliches Fleischfarbenvergleichsphotometer für den praktischen Schlach tbetrieb. Die Fleischwirtschaft, 44, 658.

Ortu A., Drews R., I966. Versuche über den Einfluss von Eisen im Futter auf die Fleischfarbe von Schnellmastkälbern. Das wirtschaftseigene Futter, 12, 35.

Pyck J., Hoste J., Gillis J., I958. Onderzoek met behulp van radioactieve tracers omtrent het verlies van sporenelementen bij de destructie van biologisch materiaal. Meded. K. vloam. Acad. 20,8.

Roy J. H. B., et al., I964. The nutrition of the veal calf. The effect of anemia and of iron and chlortetracycline supplementation on the performance of calves given large quantities of whole milk. $B r$. J. Nutr., 18, 467 .

Schmitten F., NoAck D., 1967. Über den Verlauf des Hämatokrit-Gehaltes bei Mastkälberm und seine Beeinflussung durch Fütterung und Haltung. Dt. tierärztl. Wschr., 74, I I2.

Settlemire C. T., Hibbs J. W., Conrad H. R., 1964. Basal metabolic rate, respiration rate, and certain organic weights in relation to neonatal iron deficiency anemia in dairy calves. J. Dairy Sci., 47, 875 .

Verbeke R., Martin J., I967. Contribution à l'étude de la couleur de la viande de veaux blancs. Communication $n^{\circ} 8$. Centre d'études bovines. Coupure, 235, Gent.

YeAtEs N. T. M., I g66. Iron status of calves fed an all-milk diet. Vet. Rec., 78, 547 . 